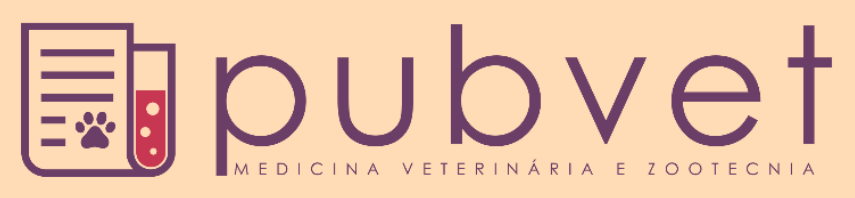

https://doi.org/10.31533/pubvet.v14n3a533.1-7

\title{
Aplicação adicional de bioestimulantes em estádio reprodutivo de feijão comum (Phaseolus vulgaris $l$.) com intuito de acréscimo na produtividade
}

\author{
Lucas Thadeu Silva Santos ${ }^{10}$, Igor Leonardo Vespucci ${ }^{2}$, Milanna Paula Cabral Nunes $^{30}$ \\ ${ }^{1}$ Engenheiro Agrônomo, Centro Universitário Montes Belos, São Luís de Montes Belos/GO - Brasil. \\ ${ }^{2}$ Doutorando, Departamento de pós-graduação em Agronegócio, Universidade Federal de Goiás - UFG - Goiânia/GO - Brasil. \\ ${ }^{3}$ Mestranda, Departamento de pós-graduação em Engenharia Agrícola, Universidade Estadual de Goiás - UEG - Anápolis/GO - Brasil \\ Autor para correspondencia, e-mail: $\underline{\text { _thadeu@hotmail.com }}$
}

\begin{abstract}
Resumo. O presente trabalho teve como objetivo avaliar a resposta produtiva do feijão em função da pulverização adicional de bioestimulantes. O delineamento utilizado foi de blocos casualizados com 6 tratamentos e 5 repetições. Foram utilizados três produtos para compor os tratamentos, sendo eles: 1 - Ácido 4-indol-3-ilbutírico $(0,05 \mathrm{~g} / \mathrm{L})$, Cinetina $(0,09$ g/L) e Ácido Giberélico (0,05 g/L); 2 - Ácido Giberélico (400 g/kg); 3 - Ácido Giberélico $(18,8 \mathrm{~g} / \mathrm{L})$ e Benziladenina $(18,8 \mathrm{~g} / \mathrm{L})$. As variáveis avaliadas consistiram em altura de planta, diâmetro de caule, número de nós por planta, número de vagens por planta, número de grãos por vagem, massa de mil grãos e produtividade. Foi realizado o Scott-Knott 5\% de probabilidade. Não houve diferença estatística significativa para as variáveis, exceto para produtividade, em que os melhores tratamentos foram 4, 5, 6. Portanto, dentre estes tratamentos o melhor devido ao custo-benefício foi o T4.
\end{abstract}

Palavras-chave. Feijoeiro, produção, reguladores de crescimento

\section{Additional application of bio-stimulants in a common bean (Phaseolus vulgaris l.) reproductive stadium with an increasing intuit in productivity}

\begin{abstract}
The present work had as objective to evaluate the productive response of the bean due to the additional spraying of biostimulants. a randomized complete block design with 6 treatments and 5 replications. Three products were used to compose the treatments: 1 - 4-Indol3-ylbutyric Acid $(0.05 \mathrm{~g} / \mathrm{L})$, Kinetin $(0.09 \mathrm{~g} / \mathrm{L})$ and Giberelic Acid $(0.05 \mathrm{~g} / \mathrm{L}) ; 2$ - Giberelic acid $(400 \mathrm{~g} / \mathrm{kg}) ; 3$ - Gibberellic acid $(18.8 \mathrm{~g} / \mathrm{L})$ and Benzyladenine $(18.8 \mathrm{~g} / \mathrm{L})$. The evaluated variables consisted of plant height, stem diameter, number of nodes per plant, number of pods per plant, number of grains per pod, mass of one thousand grains and productivity. The ScottKnott $5 \%$ probability was performed. There was no statistically significant difference for the variables, except for productivity, in which the best treatments were 4, 5 and 6 . Therefore, among these treatments the best one due to the cost-benefit was the T4.
\end{abstract}

Keywords. Bean, production, growth regulators

\section{Aplicación adicional de bioestimulantes de la etapa reproductiva del frijol común (Phaseolus vulgaris l.) para aumentar el rendimiento}

Resumen. El presente trabajo tuvo como objetivo evaluar la respuesta productiva del frijol en función de la pulverización adicional de bioestimulantes. Se utilizó un diseño de bloques completos al azar con 6 tratamientos y 5 repeticiones. Se utilizaron tres productos para componer los tratamientos: 1 - ácido 4-indol-3-ilbutírico $(0,05 \mathrm{~g} / \mathrm{L})$, cinetina $(0,09 \mathrm{~g} / \mathrm{L})$ y ácido giberélico (0,05 g/L); 2 - ácido giberélico (400 g/kg); 3 - Ácido giberélico (18,8 g/L) 
y benciladenina $(18,8 \mathrm{~g} / \mathrm{L})$. Las variables evaluadas consistieron en altura de la planta, diámetro del tallo, número de nodos por planta, número de vainas por planta, número de granos por vaina, masa de mil granos y rendimiento. Se realizó la probabilidad del 5\% de Scott-Knott. No hubo diferencias estadísticamente significativas para las variables, excepto para la productividad, donde los mejores tratamientos fueron 4, 5, 6. Por lo tanto, entre estos tratamientos, el mejor debido al costo-beneficio fue T4.

Palabras clave. Frijol, producción, reguladores del crecimiento

\section{Introdução}

O feijão comum (Phaseolus vulgaris L.) pertence à família Fabaceae, que compreende as leguminosas e está dentre os alimentos mais antigos da humanidade, sendo uma das primeiras espécies domesticadas pelo homem. É um alimento tradicional em todo o Brasil, sendo a maior fonte de proteínas da maioria dos brasileiros de classe social baixa, sendo de suma importância econômica e social para o país (Pinto, 2016). Por não possuir aminoácidos essenciais e baixo valor nutricional de proteínas, o feijão é muito consumido juntamente com o arroz. O que melhora a digestibilidade e absorção das proteínas, além de suprir os aminoácidos considerados essenciais (Pinto, 2016; Pires et al., 2006; RamírezCárdenasi et al., 2008).

No ano de 2016 foi produzida mais de 26,8 toneladas de feijão seco. Os países que mais produziram o grão foram Mianmar, Índia, Brasil e Estados Unidos, com 19,3\%; 14,5\%; 9,7\% e 4,7\% da produção mundial respectivamente ( $\underline{\mathrm{FAO}, 2017})$.

Os hormônios vegetais ou fitormônios são compostos orgânicos não sendo nutrientes de ocorrência natural, produzidos pelas plantas. Estes compostos em pequenas quantidades regulam vários processos metabólicos das plantas, promovendo, inibindo ou modificando processos morfológicos e fisiológicos dos vegetais. Eles exercem um papel, determinado, funcionando como sinais químicos específicos nas células vegetais. $\mathrm{O}$ conhecimento de locais de produção, bem como as vias de transporte, estrutura química e os mecanismos de ação dos grupos de fitormônios são de suma importância para estudos visando à alteração de respostas fisiológicas das plantas, por meio da aplicação de substâncias sintéticas ou naturais (Vieira et al., 2010).

Reguladores vegetais ou biorreculadores são substâncias sintetizadas, que, aplicadas externamente têm capacidade de ação similar aos grupos de hormônios vegetais. Retardadores vegetais são substâncias sintetizadas que tem função de inibir o crescimento do meristema subapical, retardando a alongação e a divisão celular do meristema subapical ou retardando a dominância apical. Inibidores vegetais fazem referência a substâncias naturais ou sintéticas que inibem o meristema apical (Vieira et al., 2010).

Sendo assim se questiona se será possível os fitormônios fazerem efeitos benéficos à cultura do feijoeiro. Deste modo, o presente trabalho teve como objetivo a pulverização adicional de bioestimulantes no estádio reprodutivo de feijão comum Phaseolus vulgaris L. cv. Nuance, a fim de promover maiores produtividades.

\section{Materiais e métodos}

O trabalho foi realizado na fazenda São José do Areado localizada no município de Paraúna, Goiás, sob coordenadas geográficas 16059'11.18” S, 50²3'42.33” O, com altitude de 621 metros (Köppen \& Geiger, 1928). O solo da propriedade é classificado como Latossolo vermelho (EMBRAPA, 2006). A área possui sistema de irrigação do tipo pivô central.

A cultivar utilizada foi a IAC Nuance, a cultivar é de ciclo precoce, cerca de 70 dias de cultivo, com grãos rajados de coloração creme com listras vinho claro, apresentando sementes arredondadas (elípticas) (Gonçalves, 2017). O plantio iniciou no dia 01/07/2018 com estande final de 11,3 plantas por metro linear, totalizando 226 mil plantas por hectare. Para o plantio foi utilizada uma semeadora New Holland PI 5000 com 12 linhas de plantio espaçadas em $0,5 \mathrm{~m}$ sendo semeadas aproximadamente 12 sementes por metro linear. Utilizou-se $300 \mathrm{~kg} / \mathrm{ha}$ do adubo 8-30-10 no sulco de plantio, seguindo recomendações propostas por Sousa \& Lobato (2004) em função da análise de solo no perfil de 0-20 
$\mathrm{cm}$. Sendo realizada posterior adubação de cobertura a lanço com $120 \mathrm{~kg} / \mathrm{ha}$ de cloreto de potássio, 100 $\mathrm{kg} / \mathrm{ha}$ de sulfato de amônio e $200 \mathrm{~kg} / \mathrm{ha}$ de ureia.

O delineamento foi em blocos ao acaso (DBC) com seis tratamentos (Quadro 1) e cinco repetições. Cada unidade experimental foi dimensionada com $9 \mathrm{~m}^{2}(3 \mathrm{~m} \times 3 \mathrm{~m})$, sendo utilizado seis linhas de plantio por três metros. A localização tanto dos blocos, quanto dos tratamentos, foi definida por meio de sorteio. Foram utilizados três produtos para a condução do experimento (Tabela 1).

Quadro 1. Descrição resumida dos tratamentos utilizados durante o experimento.

\begin{tabular}{|c|c|}
\hline Tratamentos & Descrição \\
\hline Tratamento 1 , Controle & Tratamento padrão da propriedade $(0,25 \mathrm{~L} / \mathrm{ha}$ do produto $1 \mathrm{em} \mathrm{V4})$. \\
\hline Tratamento 2 & $0,5 \mathrm{~L} / \mathrm{ha}$ do produto $1 \mathrm{em} \mathrm{V} 4$. \\
\hline Tratamento 3 & $\begin{array}{l}0,25 \mathrm{~L} / \mathrm{ha} \text { do produto } 1+2 \text { aplicações de } 5 \mathrm{~g} / \mathrm{ha} \text { do produto } 2 \text { (sendo a primeira em } \mathrm{R} 5 \text { e a } \\
\text { segunda } 7 \text { dias após a primeira). }\end{array}$ \\
\hline Tratamento 4 & $\begin{array}{l}\text { 0,25 L/ha do produto } 1+2 \text { aplicações de } 50 \mathrm{ml} / \mathrm{ha} \text { do produto } 3 \text { (sendo a primeira em } \mathrm{R} 5 \text { e a } \\
\text { segunda } 7 \text { dias após a primeira) . }\end{array}$ \\
\hline Tratamento 5 & $\begin{array}{l}0,25 \mathrm{~L} / \text { ha do produto } 1+2 \text { aplicações de } 50 \mathrm{ml} / \mathrm{ha} \text { do produto } 3 \text { (sendo a primeira em } \mathrm{R} 5 \text { e a } \\
\text { segunda } 7 \text { dias após a primeira) }+2 \text { aplicações de } 5 \mathrm{~g} / \mathrm{ha} \text { do produto } 2 \text { (sendo a primeira } 7 \text { dias } \\
\text { após a última aplicação do produto } 3 \text { e a segunda } 7 \text { dias após a primeira do produto } 2 \text { ). }\end{array}$ \\
\hline Tratamento 6 & $\begin{array}{l}0,5 \mathrm{~L} / \text { ha do produto } 1+2 \text { aplicações de } 50 \mathrm{ml} / \mathrm{ha} \text { do produto } 3 \text { (sendo a primeira em } \mathrm{R} 5 \text { e a } \\
\text { segunda } 7 \text { dias após a primeira) }+2 \text { aplicações de } 5 \text { g/ha do produto } 2 \text { (sendo a primeira } 7 \text { dias } \\
\text { após a última aplicação do produto } 2 \text { e a segunda } 7 \text { dias após a primeira do produto } 2 \text { ). }\end{array}$ \\
\hline
\end{tabular}

A aplicação foi realizada utilizando pulverizador costal com capacidade de $20 \mathrm{~L}$ de armazenamento de calda, com vazão aproximada de $200 \mathrm{~L}$ de cauda por hectare, utilizando bicos do tipo leque. Para a pulverização dos produtos seguiram as recomendações dos fabricantes e utilizou-se calda de 4 litros de água. Foram realizadas as aplicações do ocorreram de 24 de julho de 2018 a 26 de agosto. Todo o controle de pragas e doenças seguiram o padrão da propriedade, com o uso de herbicidas em pós emergência, inseticidas e fungicidas, para o controle e prevenção de pragas, doenças e plantas daninhas

Tabela 1. Formulação dos produtos utilizados nos tratamentos.

\begin{tabular}{lc}
\hline Produtos & Formulação \\
\hline Produto $1^{*}$ & Ácido 4-indol-3-ilbutírico $(0,05 \mathrm{~g} / \mathrm{L})$, Cinetina $(0,09 \mathrm{~g} / \mathrm{L})$ e Ácido Giberélico $(0,05 \mathrm{~g} / \mathrm{L})$ \\
Produto 2 & Ácido Giberélico $(400 \mathrm{~g} / \mathrm{kg})$ \\
Produto 3 & Ácido Giberélico $(18,8 \mathrm{~g} / \mathrm{L})$ e Benziladenina $(18,8 \mathrm{~g} / \mathrm{L})$ \\
\hline
\end{tabular}

*Único produto com recomendação pro feijão; sendo aconselhado aplicar em estádio vegetativo V4.

As variáveis avaliadas foram: altura de planta; diâmetro de caule; número de nós por planta; número de vagens por planta; número de grãos por vagem; massa de mil grãos e produtividade.

Os dados obtidos das médias das variáveis observadas foram submetidos à análise de variância $(\mathrm{P}<0,05) \mathrm{e}$ teste de Scott-Knott quando significativo, utilizando o software estatístico Sisvar 5,6. (Ferreira, 2011).

\section{Resultados e discussão}

A adição de reguladores de crescimento ao protocolo hormonal da fazenda mostrou um acréscimo na produtividade do feijoeiro. Foi observado o incremento de produtividade de alguns tratamentos em comparação ao tratamento controle. No entanto, não houve diferença significativa entre os tratamentos para a maioria das variáveis avaliadas utilizando o teste de F (Tabela 2).

Para a avaliação de altura, quanto para diâmetro de caule não foi observado diferença significativa entre os tratamentos, pelo teste de $\mathrm{F}$ a $5 \%$ de probabilidade, o que demonstra que a aplicação dos reguladores de crescimento não proporcionou aumento no porte das plantas e diâmetro do caule (Figura 1). Resultado semelhante ao observado por Castro et al. (1990) em que foi notado que a pulverização de 
giberelina na proporção de 50 ppm tenderam a apresentar altura superior de 7 a 21 dias após a aplicação, não havendo; no entanto, diferença significativa pelo teste de Tukey a 5\% de probabilidade.

Tabela 2. Médias em cm dos parâmetros avaliados, altura, diâmetro de caule, número de nós por planta, número de vagens por planta e número de grãos por vagem.

\begin{tabular}{lccccc}
\hline Tratamentos & Altura & Diâmetro & $\mathrm{N}^{\mathbf{0}}$ de nós planta & $\mathrm{N}^{\mathbf{0}}$ de vagens planta & $\mathrm{N}^{\mathbf{0}}$ de grãos vagem \\
\hline 1 & 44.17 & 4.666 & 12.332 & 7.032 & 3.64 \\
2 & 43.666 & 4.6 & 13.234 & 8.734 & 3.544 \\
3 & 43.766 & 4.8 & 12.168 & 6.532 & 3.708 \\
4 & 44.9 & 4.7 & 12.834 & 8.466 & 3.79 \\
5 & 44.8 & 4.866 & 13.868 & 8.734 & 3.764 \\
6 & 45.002 & 4.9 & 12.966 & 7.866 & 3.876 \\
\hline
\end{tabular}

As médias não se diferiram estatisticamente pelo teste de Scott-Knott.

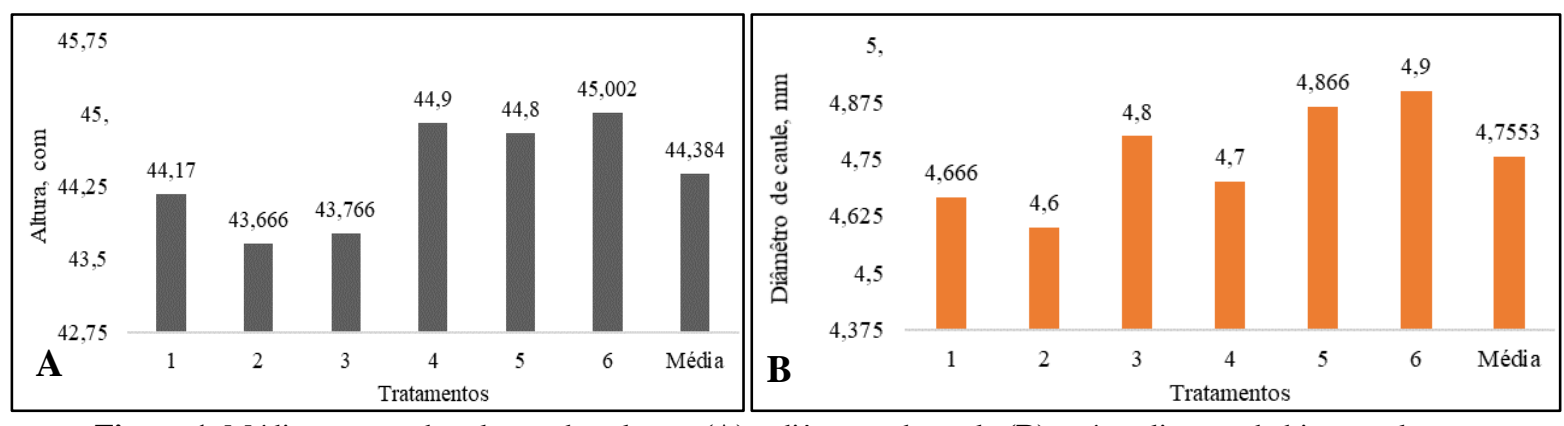

Figura 1. Médias em cm das alturas das plantas (A) e diâmetro de caule (B) após aplicação de bioestumlantes.

Para o número de nós por planta foi observado semelhança estatística para todos os tratamentos, não sendo significante para os testes de $\mathrm{F}$ a $5 \%$ de probabilidade, portanto, os tratamentos não proporcionaram maior área foliar e número de nós produtivos. Entretanto, o tratamento 5 apresentou um leve aumento no número médio de nós perante os demais tratamentos, apresentando 13,868 nós em média por planta (Figura 2A). Almeida et al. (2014) em sua pesquisa, observou resultado parecido, ao utilizar bioestimulantes no tratamento de sementes, em aplicação foliar em estádio vegetativo (V4) e reprodutivo (R5), não sendo observada significância entre os tratamentos utilizandos, apesar de um leve aumento no número de nós ao aplicar o bioestimulante em tratamento de sementes + R5.

$\mathrm{O}$ número de vagens por planta, número de grãos por vagem e massa de mil grãos não diferiram estatisticamente pelos testes F (Figura 2A, 2B e 2C). Resultado condizente foi observado por Alleoni et al. (2009) e Almeida et al. (2014) que constatou em sua pesquisa com a aplicação de regulador de crescimento em tratamento de sementes e foliar, que houve um aumento no número de vagens, grãos por vagem, não havendo, entretanto, diferença significativa. Bernardes et al. (2010), em seu trabalho obteve resultados semelhantes utilizando regulador de crescimento no tratamento de sementes e em aplicação foliar, em feijão cultivado em palhada de diferentes culturas antecessoras de cobertura, e também observou um ligeiro acréscimo no número de vagens, todavia, não foram observadas diferenças significativas entre os tratamentos. Dados obtidos por Lana et al. (2009), não observou diferenças significativas entre os tratamentos, sendo estes, com a utilização de reguladores de crescimento tanto no tratamento de sementes, quanto em aplicação foliar.

A produtividade das variáveis analisadas foi significativamente diferente, ficando evidente que os tratamentos 4, 5 e 6 foram diferentes dos demais tratamentos; porém, foram semelhantes entre si (Tabela 3).

As médias de produtividade dos tratamentos foram expressas em duas unidades distintas, para uma melhor visualização dos resultados, sendo os tratamentos 4,5 e 6 com maiores médias em quilogramas por hectare, com $2.598 \mathrm{~kg} / \mathrm{ha}, 2.624 \mathrm{~kg} / \mathrm{ha} \mathrm{e} 2.650 \mathrm{~kg} / \mathrm{ha}$, respectivamente (Figura $3 \mathrm{~A}$ ) e também em sacas por hectare, com 43,298 scs/ha, 43,734 scs/ha e 44,166 scs/ha, respectivamente (Figura 3B).

Este acréscimo na produtividade pode ser explicado ao observar que mesmo não havendo resultados significativos estatisticamente, o número de grãos por vagem foi ligeiramente maior entre os tratamentos 
4, 5 e 6, que obtiveram maiores médias de produtividade. Bem como no número de vagens por planta, onde os tratamentos 5, 2, 4 e 6 obtiveram as maiores médias, apresentando 8.734, 8.734, 8.466 e 7.866, respectivamente. O que mostra que a aplicação dos reguladores de crescimento (Produtos 2 e 3 ) em estádio reprodutivo no feijoeiro contribui para o aumento de produtividade. Este resultado condiz com o observado por Bertolin et al. (2010) que encontraram em sua pesquisa, ao analisar a aplicação de bioestimulantes em soja, médias maiores de produção em aplicações no estádio reprodutivo R1 e no tratamento de sementes + R5.

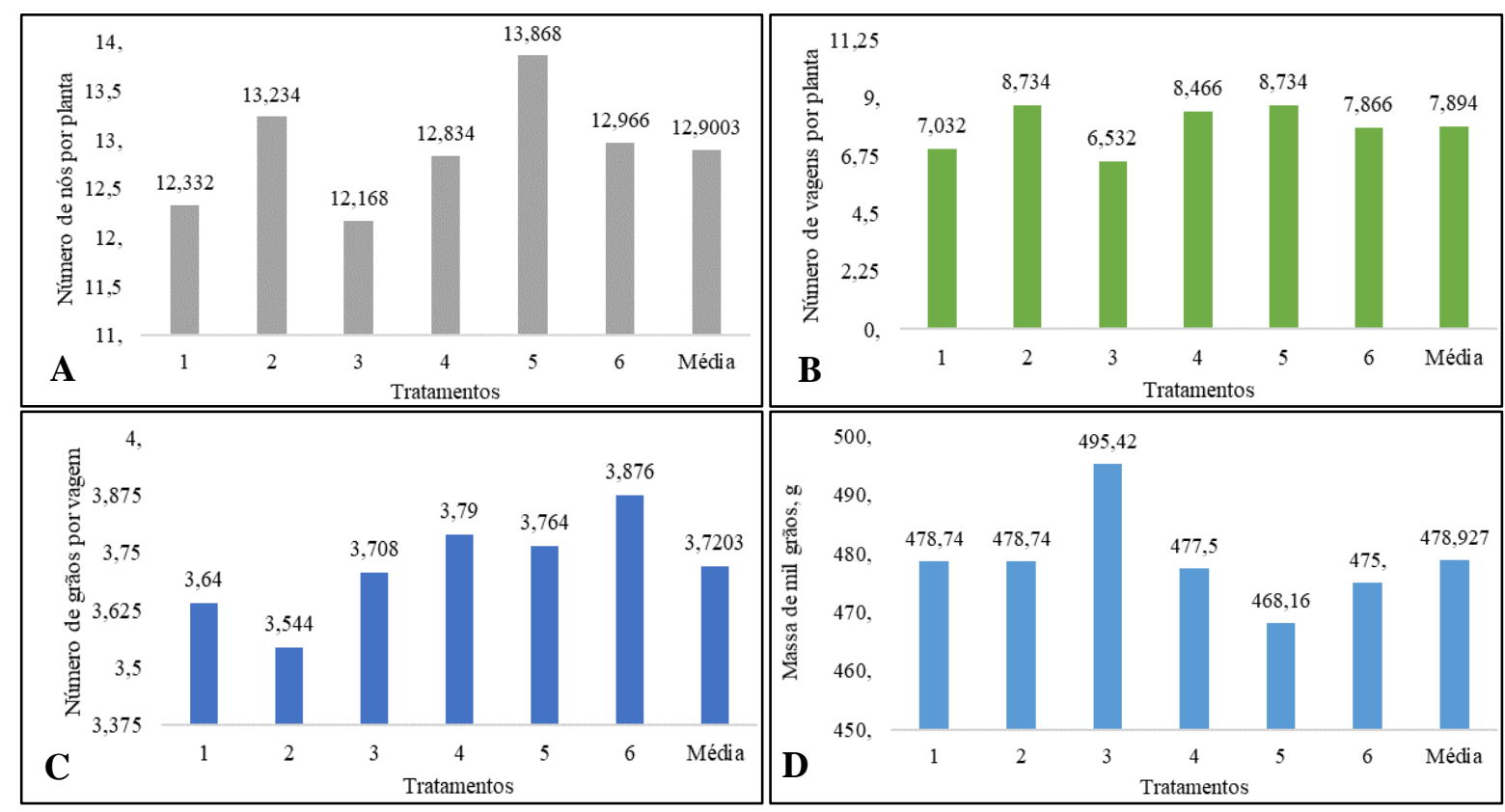

Figura 2. Médias de números de nós por planta (A), número de vagens por planta (B), número de grãos por vagem $(\mathbf{C})$, massa de mil grãos (D) em função dos tratamentos com bioestimulantes.

Tabela 3. Médias harmônicas para os parâmetros avaliados: Massa de mil grãos, Produtividade (kg/ha) e produtividade em sacas $(60 \mathrm{~kg}) / \mathrm{ha}$.

\begin{tabular}{lclclcc}
\hline Tratamentos & Massa de Mil grãos & & Produtividade (kg/ha) & & Produtividade (scs/ha) \\
\hline 1 & 478.74 & $\mathrm{a}$ & 2116 & $\mathrm{~b}$ & 35.268 & $\mathrm{~b}$ \\
2 & 478.74 & $\mathrm{a}$ & 2256 & $\mathrm{~b}$ & 37.6 & $\mathrm{~b}$ \\
3 & 495.42 & $\mathrm{a}$ & 2030 & $\mathrm{~b}$ & 33.834 & $\mathrm{~b}$ \\
4 & 477.5 & $\mathrm{a}$ & 2598 & $\mathrm{a}$ & 43.298 & $\mathrm{a}$ \\
5 & 468.16 & $\mathrm{a}$ & 2624 & $\mathrm{a}$ & 43.734 & $\mathrm{a}$ \\
6 & 475 & $\mathrm{a}$ & 2650 & $\mathrm{a}$ & 44.166 & $\mathrm{a}$ \\
\hline Média & 478.93 & & 2379.00 & & 39.65 &
\end{tabular}

Médias seguidas de mesma letra não se diferiram estatisticamente pelo teste de Scott-Knott.
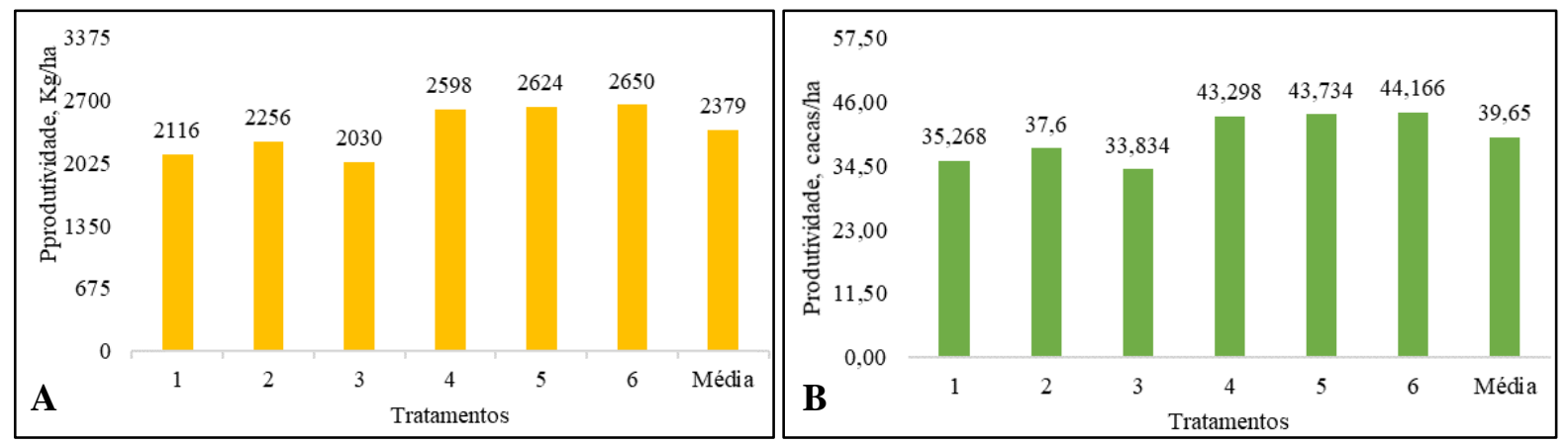

Figura 3. Médias de produtividade (expressa em quilogramas por hectare) (A) e de produtividade (expressa em sacas por hectare) (B) em função dos tratamentos com bioestimulantes. 
Ávila et al. (2010) não observaram resultado semelhante, em seu estudo que realizou a aplicação de bioestimulante em diferentes estádios fenológicos do feijoeiro, com e sem a adição de cálcio e boro, na presença e ausência de irrigação, não encontrou diferença significativa entre os tratamentos. Por outro lado, Abrantes (2008) observou que uma dosagem maior, bem como, a aplicação em estádio fenológico reprodutivo proporcionou maior produtividade para duas cultivares de feijão carioca, IAC Apuã e Carioca Precoce, obtendo assim resultados estatisticamente diferentes.

\section{Considerações finais}

Nas condições experimentais, evidencia-se que o uso de bioestimulantes apenas no estádio vegetativo quanto no reprodutivo não proporcionou diferenças significativas na morfologia e arquitetura das plantas, havendo, entretanto, um acréscimo em produtividade ao aplicar os Produtos 2 e 3 em fase reprodutiva no feijoeiro. Sendo a aplicação dos dois produtos em adição a dose máxima recomendada do Produto 1 o tratamento com maior média de produtividade (Tratamento 6), todavia, por não se diferir estatisticamente do Tratamento 4, onde apenas o produto 2 foi aplicado em adição a dose mínima do produto 1, o Tratamento 4 se mostrou mais vantajoso, por economizar em quantidade de produtos, dose e aplicações, diminuindo assim o custo do manejo.

\section{Referências bibliográficas}

Abrantes, Fabiana Lima. (2008). Efeito de bioestimulante sobre a produtividade fisiológica de dois cultivares de feijão cultivados no inverno. 68 p. Dissertação (Mestrado em Agronomia) Faculdade de Engenharia - UNESP, Ilha Solteira.

Alleoni, B., Bosqueiro, M., \& Rossi, M. (2009). Efeito dos reguladores vegetais de Stimulate ${ }^{\circledR}$ no desenvolvimento e produtividade do feijoeiro (Phaseolus vulgaris L.). Ciências Exatas e Da Terra, Ciências Agrárias e Engenharias, 6(1), 23-35.

Almeida, A. Q., Soratto, R. P., Broetto, F., \& Cataneo, A. C. (2014). Nodulação, aspectos bioquímicos, crescimento e produtividade do feijoeiro em função da aplicação de bioestimulante. Semina Ciências Agrarias, 1, 77-88.

Ávila, M. R., Barizão, D. A. O., Gomes, E. P., Fedri, G., \& Albrecht, L. P. (2010). Cultivo de feijoeiro no outono/inverno associado à aplicação de bioestimulante e adubo foliar na presença e ausência de irrigação. Scientia Agraria, 11(3), 221-230.

Bernardes, T. G., Silveira, P. M., \& Mesquita, M. A. M. (2010). Bernardes, T. G., Silveira, P. M., Mesquita, M. A. (2010). Produtividade Do Feijoeiro Irrigado Devido A Reguladoresde Crescimento E Culturas Antecessoras De Cobertura. Bragantia, Campinas, v.69, n.2, p.371-375. Bragantia, 69(2), 371-375.

Bertolin, D. C., Sá, M. E., Arf, O., Furlani Junior, E., Colombo, A. S., \& Carvalho, F. L. B. M. (2010). Aumento da produtividade de soja com a aplicação de bioestimulantes. Bragantia, 69(2), 339-347.

Castro, P. R. C., Appezzato, B., Lara, C., Pelissari, A., Pereira, M., Medina, M., Bolonhezi, A. C., \& Silveira, J. A. G. (1990). Ação de reguladores vegetais no desenvolvimento, aspectos nutricionais, anatômicos e na produtividade do feijoeiro (Phaseolus vulgaris cv. Carioca). Anais Da Escola Superior de Agricultura Luiz de Queiroz, 47(1), 11-28.

EMBRAPA. (2006). Sistema brasileiro de classificação de solos. In Embrapa (Vol. 412).

FAO. (2017). Statistical Yearbook (Vol. 1, Issue 1). Food and Agriculture Organization of the United Nations.

Ferreira, D. F. (2011). SISVAR: A Computer Statistical Analysis System. Ciência e Agrotecnologia, 35(6), 1039-1042. https://doi.org/http://dx.doi.org/10.1590/S1413-70542011000600001.

Gonçalves, C., Aguiar, A.T.E., Capanema, L.M. (2018). Cultivares IAC. 2017. Disponível em: $<$ http://oagronomico.iac.sp.gov.br/?p=854>. Acesso em: 30 set.

Köppen, W., \& Geiger, R. (1928). Klimate der Erde. Gotha: Verlag Justus Perthes. Wall-Map $150 \mathrm{~cm} \times 200 \mathrm{~cm}$.

Lana, R. M. Q., Lana, A. M. Q., Gozuen, C. F., Bonotto, I., \& Trevisan, L. R. (2009). Aplicação de reguladores de crescimento na cultura do feijoeiro. Bioscience Journal, 25(1), 13-20. 
Pinto, J. V. (2016). Propriedades físicas, químicas, nutricionais e tecnológicas de feijões (Phaseolus vulgaris $L$.) de diferentes grupos de cor.

Pires, C. V., Oliveira, M. G. de A., Rosa, J. C., \& Costa, N. M. B. (2006). Qualidade nutricional e escore químico de aminoácidos de diferentes fontes protéicas. Food Science and Technology, 26(1), 179187.

Ramírez-Cárdenasi, L., Leonel, A. J., \& Costa, N. M. B. (2008). Efeito do processamento doméstico sobre o teor de nutrientes e de fatores antinutricionais de diferentes cultivares de feijão comum. Food Science and Technology, 28(1), 200-213.

Sousa, D. M. G., \& Lobato, E. (2004). Cerrado: correção do solo e adubação. Embrapa Cerrados, 1, 416.

Vieira, E. L., de Souza, G. S., dos Santos, A. R., \& dos Santos Silva, J. (2010). Manual de fisiologia vegetal. Edufma.

Recebido: 03 de novembro, 2019.

Aprovado: 12 de dezembro, 2019.

Publicado: 23 de abril, 2020.

Licenciamento: Este artigo é publicado na modalidade Acesso Aberto sob a licença Creative Commons Atribuição 4.0 (CC-BY 4.0), a qual permite uso irrestrito, distribuição, reprodução em qualquer meio, desde que o autor e a fonte sejam devidamente creditados. 